\title{
$1 \quad$ Local and regional specialization in plant-pollinator networks
}

3 Daniel W. Carstensen ${ }^{1,4}$, Kristian Trøjelsgaard ${ }^{2}$, Jeff Ollerton ${ }^{3}$ and Leonor Patricia C. Morellato ${ }^{1}$ 4

$5{ }^{1}$ São Paulo State University (UNESP ), Inst. of Biosciences, Dept of Botany, Phenology Lab, Rio

6 Claro, São Paulo, Brazil. Email: daniel.carstensen@gmail.com. Orcid id: orcid.org/0000-0003-

$7 \quad 3697-1688$

$8 \quad{ }^{2}$ Department of Chemistry and Bioscience, Aalborg University, Aalborg, Denmark

$9{ }^{3}$ Faculty of Arts, Science and Technology, University of Northampton, Avenue Campus, NN2 6JD, 10 U.K.

$11{ }^{4}$ Center for Macroecology, Evolution and Climate, Natural History Museum of Denmark, 12 University of Copenhagen, Universitetsparken 15, DK-2100 Copenhagen $\varnothing$, Denmark. 


\section{Abstract}

Specialization of species is often studied in ecology but its quantification and meaning is disputed. More recently, ecological network analysis has been widely used as a tool to quantify specialization, but here its true meaning is also debated. However, irrespective of the tool used, the geographic scale at which specialization is measured remains central. Consequently, we use data sets of plant-pollinator networks from Brazil and the Canary Islands to explore specialization at local and regional scales. We ask how local specialization of a species is related to its regional specialization, and whether or not species tend to interact with a non-random set of partners in local communities. Local and regional specialization were strongly correlated around the 1:1 line, indicating that species conserve their specialization levels across spatial scales. Furthermore, most plants and pollinators also showed link conservatism repeatedly across local communities, and thus seem to be constrained in their fundamental niche. However, some species are more constrained than others, indicating true specialists. We argue that several geographically separated populations should be evaluated in order to provide a robust evaluation of species specialization.

Keywords: community, scale, spatial variation, partner fidelity, link conservatism 


\section{Introduction}

The Earth's biodiversity is shaped by a plethora of interactions between species that may range from being relatively specialized to relatively generalized, and the exact nature of these interactions depend upon a variety of ecological and evolutionary factors. Many of these types of interactions are common and ecologically vital; pollination by animals for example is the norm for an estimated 87.5\% of the c. 352,000 species of flowering plants (Ollerton et al. 2011). Extensive work based on visitation data between plants and pollinators indicate that such interactions tend to be more generalized than previously thought, even for tropical systems (Ollerton and Cranmer 2002, Schleuning et al. 2012, Waser et al. 1996). However, recent studies, considering visitation frequency and service effectiveness, suggest stronger fidelity between interaction partners and that binary visitation data might overestimate generalization levels (King et al. 2013, Rosas-Guerrero et al. 2014 - though see Ollerton et al. 2015). Furthermore, several studies indicate that species can appear as generalists while actually being composed of specialist populations, or even generalist populations composed of specialist individuals (Araujo et al. 2008, Bolnick et al. 2002, Devictor et al. 2010, Dupont et al. 2011, Fox and Morrow 1981, Tur et al. 2014). Such studies suggest that phylogenetic and geographic scale are highly relevant when studying specialization, and sampling of multiple populations is necessary to reliably determine the niche breath of a species to account for cross-community variation (Carstensen et al. 2014, Fox and Morrow 1981, Ollerton et al. 2007, Ollerton et al. 2009, Poisot et al. 2015, Trøjelsgaard et al. 2015).

The fundamental taxonomic niche of a species describes the set of potential interaction partners as determined by functional traits (Junker et al. 2013). Conversely, the realized taxonomic niche is the subset of species within a given community with which the focal species interacts. Thus, while functional traits do not necessarily enable us to predict the occurrence of a given interaction, they do partly define the set of possible interactions (Morales-Castilla et al. 2015) and 
trait complementarity does seem to consistently govern general interaction patterns within communities (Carstensen et al. 2016). The fundamental and realized niche concepts are related to specialization at the level of species and populations respectively, and the fundamental niche is seldom fully realized in any single local community because of differences in relative abundances, species composition and other biotic and abiotic factors (Burkle et al. 2016, Carstensen et al. 2014, Trøjelsgaard et al. 2015).

Here, we explore species specialization across interacting plants and pollinators within two contrasting regions: Brazilian campo rupestre, or rupestrian grasslands, and the Canary Islands. Both datasets consist of seven spatially separated community-level plant-pollinator networks, sampled in a standardized way within their regions. Using these data we explore the relationship between local and regional specialization, and investigate whether this relationship differ between these two contrasting study regions, assuming the ecological specialization framework (Armbruster 2017). Specifically we ask: 1) Are local and regional measures of specialization correlated? 2) Do species interact locally with a non-random subset of the available species in the community?

We expect that the specialization level of a species is conserved across populations, but that the identity of interaction partners might change. If so, species would appear more generalized at the regional level compared to the local level.

\section{Methods}

Study sites and field observations

Data were collected in October-December 2012 from seven sites of rupestrian grasslands, or campo rupestre, in the National Park of Serra do Cipó, SE Brazil (Carstensen et al. 2014, 2016). Campo rupestre is a species-rich vegetation of mostly shrubs and herbs associated with rocky outcrops surrounded by sandy and stony grasslands (Silveira et al. 2016). Sites were 1.4-8.5 km 
apart within an altitudinal range of 1073-1260 m a.s.l. One site was sampled per day with a weekly rotation among sites. Attempting to observe all flowering plant species in each site, plant individuals were observed in 15 min intervals (totalling 252 hours of observation), recording all visitors touching the reproductive floral parts (for more details see Carstensen et al. 2014). For each 15-min interval randomly selected plant individuals were observed, and if individuals of the same species were in close proximity of each other they were often surveyed simultaneously. Flower visitors were collected for taxonomic identifications by experts. For each site, the data were summarized in a quantitative interaction matrix expressing the frequency of interactions between pairs of plants and pollinators. This resulted in seven spatially separated networks with a total of 101 plant species and 201 pollinator species.

Data were also collected from five islands within the Canarian archipelago (El Hierro, La Gomera, Tenerife (two sites), Gran Canaria and Fuerteventura) and a single site in Western Sahara located at the West African coast close to the Canary Islands (Trøjelsgaard et al. 2013). In total this gave us seven spatially separated pollination networks from habitats characterized as semi-arid and dominated by shrubs. The Canary Island networks were 53-455 km from each other and separated by ocean, except the two sampling sites at Tenerife, which, conversely, were separated by El Pico del Teide (3718 $\mathrm{m}$ a.s.1.). All flowering perennial plant species were surveyed for flower visitors in intervals of 15 min (totalling 296 hours of observation) in January-March 2010 through flowerbased focal observations in a similar way as for the Brazilian data set (for more details see Trøjelsgaard et al. 2013). Most plant species were observed in 15-min intervals approximately 8 times $($ mean $=7.0$, Std. dev. $=1.8)$. Randomly selected individuals were preferably chosen for each of the individual 15-min surveys, although low abundance of some species precluded this practice. Sometimes multiple individuals of the same species were surveyed simultaneously depending on their spatial aggregations, and also depending on the total number of flowers per individual. 
Approximately 1300 flower visitors were collected for taxonomic verifications by entomological experts, allowing species identification of many pollinators, while some were grouped as morphospecies. Overall we scored interactions between 39 plant species and 249 pollinator species and, similar to the Brazilian dataset, all seven networks from the Canary Islands were quantitative interaction matrices expressing the frequency of interactions between plants and pollinators.

\section{Specialization and interaction richness}

For each plant and pollinator species observed in more than one site, we defined a metaweb of species $s$ (Dunne 2006) as the network consisting of all species occurring in the sites in which $s$ occurs, as well as all the interactions between them. In other words, it is the regional network of interactions for species $s$ and its co-occurring species. We quantified specialization using the index $d_{s}^{\prime}$, which describes the deviation of interactions from what would be expected under neutral conditions. Instead of counting the number of interaction partners, $d_{s}^{\prime}$ measures the exclusiveness of a species' interactions and is insensitive to variation in observation frequencies across species (Blüthgen et al. 2006). Specialization, $d_{s}^{\prime}$, was calculated using the $d f u n$ function in the package 'Bipartite' in R (Dormann 2011); and for all species we calculated both a regional specialization level using the metaweb of species $s\left(d_{s, \text { meta }}^{\prime}\right)$ as well as an average local specialization level using the local networks in which species $s$ occurred $\left(d_{s, l o c a l}^{\prime}\right)$.

We tested the correlation between local and regional specialization using the Pearson correlation coefficient. Through simple linear regression, we further tested whether the regional specialization was affected by the number of sites in which a species occurred, or rather, the number of potential interaction partners with which it co-occurred across the region.

To quantify if regional interaction richness of a species differed from what could be expected if species interacted randomly with the available set of partners, we first calculated the 
regional degree for each species, $\gamma_{s}$, which is the total number of different interaction partners across all sites in which the species was observed (i.e. the degree in the metaweb for species $s$ ). We then compared the empirical $\gamma_{s}$ with a null model where the identity of partners in the local networks was randomized while conserving the local number of interactions for each species. New interaction partners were drawn with a probability proportional to their abundance (measured as total number of interactions, i.e. the marginal sums in the quantitative interaction matrix) among all potential partner species in a local community, and random partners were drawn with replacement until the empirical number of interactions was reached. In this way, the total number of local interactions (i.e. the marginal sum) was retained, but the regional degree was allowed to change as species were allocated new interaction partners among all potential partners at a local site. This procedure was repeated 1000 times for each species, which allowed us to calculate a mean $\left(\gamma_{s, \text { random }}\right)$ and standard deviation $\left(S D_{\text {random }}\right)$ for each species. Subsequently we compared empirical regional species degrees $\left(\gamma_{s}\right)$ with the randomized regional degrees $\left(\gamma_{s, \text { random }}\right)$ by using z-scores $\left[\left(\gamma_{s}-\right.\right.$ $\left.\left.\gamma_{s, \text { random }}\right) / S D_{\text {random }}\right]$. If the $\mathrm{z}$-score was less than -1.96 , or larger than 1.96 , the difference between the empirical and random regional degree was deemed statistically significant. Moreover, negative values suggest that a species is more constrained in its choice of interaction partners than if partner identities were determined solely by availability (abundance) (we call this 'link conservatism'), while positive values suggest that the species is more opportunistic than expected (we call this 'link opportunism'). By incorporating species abundance as a way of selecting partners in the null-model, the procedure leans towards the neutral perspective which assumes that interactions between species is largely determined by species abundance (see e.g. Canard et al. 2014, Poisot et al. 2015). It is important to note that the null-model ignores phenological or morphological constraints (e.g. Olesen et al. 2011), and therefore may overestimate the availability of potential partners. 
Finally, we tested for a correlation between the z-scores (link conservatism) and $d_{s, \text { meta }}^{\prime}$ (the regional specialization, or exclusiveness) in order to examine how the two measurements coincide. While $d_{s, \text { meta }}^{\prime}$ measures the deviation of interactions from neutrality based on the availability of partners in the metaweb, the z-scores measure the deviation of the interactions in the metaweb from a null model were each local network is randomized before aggregating the metaweb. These two measures thus quantify specialization, or conservatism, from slightly different approaches and should be negatively correlated. All analyses were done in R, version 3.2.0 (R Development Core Team 2008).

\section{Results}

Regional $\left(d_{s, \text { meta }}^{\prime}\right)$ and local $\left(d_{s, \text { local }}^{\prime}\right)$ specialization were significantly and strongly correlated in the Brazilian networks for both plants and pollinators (Pearson correlation, $r=0.76$ and 0.83 respectively, $\mathrm{p}<0.001$, Fig. 1A) as well as in the Canary Islands networks (Pearson correlation, $\mathrm{r}=$ 0.76 and 0.89 respectively, $\mathrm{p}<0.001$, Fig. 1B). In Brazil, the number of co-occurring species (i.e. the number of potential interaction partners in the metaweb) had no effect on regional specialization values for plants ( $\mathrm{p}>0.7, \mathrm{R}^{2}<0.01$ ), but correlated significantly with regional specialization of pollinators, albeit with a very poor fit $\left(\mathrm{p}<0.05, \mathrm{R}^{2}=0.05\right.$, indicated by size of data points in Fig. 1A). In the Canary Islands the number of co-occurring species had no effect on regional specialization for neither pollinators nor plants $\left(\mathrm{p}>0.2, \mathrm{R}^{2}<0.01\right)$. The null model analysis showed similar results for the Brazilian and Canary Islands networks (Fig. 2). In both systems, the majority of the plant species showed link conservatism because $81 \%$ (Brazil) and 57\% (Canary Islands) of the plants had significantly fewer regional interaction partners than expected if partner identity were determined solely by availability (abundance). That is, these species tend to conserve their interaction partners across sites. The majority of the pollinators also 
showed link conservatism in Brazil but not in the Canary Islands as 58\% and $46 \%$ of the pollinators, respectively, had significantly fewer regional interaction partners. Finally, the pollinators deviation from randomness (i.e. the z-scores, with negative and positive values being suggestive of link conservatism and opportunism, respectively) correlated significantly and negatively with regional specialization in both Brazil (Pearson correlation, $r=-0.34, p<0.001$ ) and the Canary Islands (Pearson correlation, $r=-0.30, \mathrm{p}=0.009$ ), while the relationship where nonsignificant for plants in both Brazil (Pearson correlation, $r=-0.25, \mathrm{p}=0.14$ ) and the Canary Islands (Pearson correlation, $\mathrm{r}=0.16, \mathrm{p}=0.50)($ Fig. 3).

\section{Discussion}

The results from the two regions were surprisingly similar. Local and regional specialization were strongly correlated in both regions. Plants were generally more specialized than pollinators (Fig. 1) despite a general trend of having more interaction partners (Fig. 2). A correlation between local and regional specialization was expected, however, a strong correlation close to 1:1 indicates that local and regional specialization, in terms of $d_{s}^{\prime}$, is largely interchangeable, and that species in plantpollinator networks are consistent in their specialization level across scales. By aggregating the specialization level across geographically separated sampling sites, the current procedure attempted to capture the differences in interactions that can be found between populations and individuals (see e.g. Araujo et al. 2008, Tur et al. 2014). In the Canary Islands the geographical distance between sites varied from 52 to $456 \mathrm{~km}$, while the distance in Brazil varied between 1.4 and $8.5 \mathrm{~km}$. Yet, the rocky outcrops found in the campo rupestre, within which the surveys were done in Brazil, can to some extent be seen as isolated habitat patches within a matrix of grassland, and the actual isolation between the populations is likely larger than the geographical distances dictates. However, we cannot preclude that increasing the geographical extent so that we covered even more distant 
populations, especially in Brazil, would lead to different results. Nevertheless, it seems encouraging that the relationship between local and regional specialization were almost identical when comparing the Brazilian and Canarian communities, which suggest that this pattern might actually be a general trend. More studies from different communities are needed, however, to explore the generality of this finding.

Larger distances and increased isolation between sites could result in local populationspecific adaptations within species, and thus translate into a larger inter-island variability in interaction partners, which ultimately would result in higher opportunism at the regional scale communities also differ in a number of other aspects. For example, across the sampling sites the ratio of pollinators:plants were on average $4.4 \pm 1.0$ (mean $\pm \mathrm{SD})$ in the Canary Islands, and $2.6 \pm 0.4$ in Brazil. A lower diversity of plants in the Canarian communities may hamper the pollinators potential to show a strong fidelity across sites when compared with a null model. However, if partner diversity was the sole explanation, we would expect the Canarian plants to show a stronger fidelity as they have more partners to choose from and, therefore, a larger potential to deviate from conservatism diminishes. The plant communities were on average more similar across the Canary 
\pm 0.14 ), while the pollinator communities on average were more similar in Brazil (average Sorensen similarity across sites $\pm \mathrm{SD}$, Canary Islands $=0.24 \pm 0.12$; Brazil $=0.43 \pm 0.06$ ). Thus, while the high turnover of pollinators at the Canary Island might explain why a lower percentage of the plants show significant link conservatism, this does not explain the patterns observed for the pollinators. Finally, the studied communities also differ in e.g. species composition, climate conditions, and other biotic and abiotic aspects, which makes it difficult to isolate a single common mechanism as responsible for the difference in link conservatism between the Canarian and Brazilian communities.

Overall, for specialist species the foraging and interaction choices are most likely a consequence of trait complementarities, while generalists could be expected to forage or interact mainly according to relative abundances of potential partners. Link conservatism should therefore be higher for specialist species, as we have confirmed (Fig. 3). Indeed, as we argue below, link conservatism and $d_{s}^{\prime}$ should be evaluated together to reliably indicate true specialists.

Our results show that most species have significantly fewer regional interactions than expected based on our null model. Thus, at the local scale species are constrained in their fundamental niche, indicating innate restrictions in their interaction partners, likely because of trait complementarity (Olesen et al. 2011, Santamaria and Rodríguez-Girones 2007). Species with the largest negative $\mathrm{z}$-values tend to interact repeatedly with the same species across sites, and they therefore show high link conservatism (see also Trøjelsgaard et al. 2015). This indicates stronger constraints on the fundamental niche. Even within the species that show significant link conservatism $(\mathrm{z}$-score $<-1.96)$ some species show much stronger constraints, indicating true specialists. Interestingly, the level of conservatism seems unrelated to regional interaction richness $\left(\gamma_{s}\right)$ (Fig. 2). We argue that labeling a species as specialist is not necessarily dependent only upon it having few interaction partners, but that interactions are repeatedly formed with the same subset of 
species, and thus showing a strong signal of link conservatism. This might add another dimension to the discussion about species specialization vs. generalization (Ollerton et al. 2007, Rosas-Guerrero et al. 2014, Schleuning et al. 2012, Waser 2006), as the categorization as specialist or generalist might be strongly dependent upon the spatial scale at which species are investigated. For pollinators there was a significant correlation between z-scores and the measurement of regional specialization $\left(d_{s, \text { meta }}^{\prime}\right)$ in both Brazil and the Canary Island, which further supports the designation of true specialists. It is here important to re-emphasize that our null model was framed around the neutral theory suggesting that biotic interaction to a large extent is governed by species abundance (Canard randomness also occurred among species having many regional interaction partners, where there is et al. 2014, Poisot et al. 2015). By ignoring any phenological and morphological constraints we may overestimate the availability of potential partners, and some species may appear more conservative than if such constraints were also taken into account. Importantly, however, large deviation form little evidence of phenological and morphological constraints, as they interact with a large proportion of the available partners. Therefore, incorporating such constraints would probably have minimal impact for at least some of the species. Still, with good phenological resolution and detailed morphological trait data the impact of these constraints could be explored further. Also, our approach could be further applied to investigate the role of floral traits in defining visitation fidelity, revisiting the discussion on the generalization in pollination system towards the tropics (Ollerton et al. 2009, Rosas-Guerrero et al. 2014, Schleuning et al. 2012), with consequences for plant reproductive success and adaptation to environmental changes.

The interaction richness of a species is ultimately limited by the availability of potential partners at a given site, which, in turn, is determined by functional traits (Ibanez et al. 2016). However, according to our results, it would be sensible to distinguish between niche breath and specialization, because link conservatism (z-scores) and niche breath (regional interaction 
richness) were not correlated (Fig. 2). In some cases, species with wider niches might repeatedly

271 choose the same partners across populations while species with narrower niches exert a random

272 pattern, indicating specialists and generalists, respectively. Thus, we argue that measures of local

273 and regional specialization, together with a null model approach, can provide a robust evaluation of

274 species specialization by including information on whether the identity of interaction partners

275 change across local populations.

276

277 Acknowledgements

278 We thank the Cedro Company, ICMBio and Reserva Vellozia for permission to work in their

279 protected areas. DWC is grateful to Nathália Miranda and Otto Carstensen for their contributions.

$281 \quad$ Funding

282 This work was funded by São Paulo Research Foundation (FAPESP) grants \#2011/22635-2 and

283 \#2014/01594-4 to DWC, \#2010/51307-0 to LPCM and Visiting Research grant \#2013/14442-5 to

284 JO, the Danish Council for Independent Research | FNU \#109751 to DWC and CNPq research 285 productivity grant to LPCM.

286

287 Author contributions

288 The first and second author contributed equally to this paper.

289 
Figure 1. Mean local specialization $\left(d_{s, \text { local }}^{\prime}\right)$ and regional specialization $\left(d_{s, \text { meta }}^{\prime}\right)$ are strongly positively correlated in both Brazil and the Canary islands. Plants and pollinators are represented by blue and red points, respectively. The size of the data points indicate the number of co-occurring species (i.e. the number of potential interaction partners in the metaweb). Note that plants are generally more specialized than pollinators.

Figure 2. Null model analysis on expected regional interaction richness vs. observed regional interaction richness $\left(\gamma_{\mathrm{s}}\right)$. A z-score below -1.96 means that the species has significantly fewer regional interaction partners than expected from random (link conservatism), and colored data points fall below this threshold. Density curves indicate the distribution of points across the spectra.

300 Note that plants generally have more interaction partners (higher interaction richness) than 301 pollinators. Plants and pollinators are represented by blue and red points, respectively.

Figure 3. Z-scores and regional specialization $\left(d_{s, \text { meta }}^{\prime}\right)$ show significantly negative correlations for 304 pollinators in both Brazil $(r=-0.34, p<0.001)$ and the Canary Islands $(r=-0.30, p=0.009)$. These 305 are highlighted with trend lines. For the plant species, however, the relationships were non306 significant in both Brazil and the Canary Islands and trend lines have been omitted. Plants and 307 pollinators are represented by blue and red points, respectively. 
310 Armbruster, W. S. (2017), The specialization continuum in pollination systems: diversity of 311 concepts and implications for ecology, evolution and conservation. Functional Ecology, 31: 88312 100. doi: $10.1111 / 1365-2435.12783$

313 Araujo, M. S., et al. 2008. Network analysis reveals contrasting effects of intraspecific competition 314 on individual versus population diets. - Ecology 89: 1981-1993.

315 Blüthgen, N., et al. 2006. Measuring specialization in species interaction networks. - BMC ecology 316 6: 1-12.

317 Bolnick, D. I., et al. 2002. Measuring individual-level resource specialization. - Ecology 83: 29363182941.

Burkle, L. A., et al. 2016. The beta-diversity of species interactions: Untangling the drivers of geographic variation in plant-pollinator diversity and function across scales. - Am J Bot 103: 118128.

Canard, E.F., Mouquet, N., Mouillot, D., Stanko, M., Miklisova, D. \& Gravel, D. (2014) Empirical Evaluation of Neutral Interactions in Host-Parasite Networks. The American Naturalist, 183, 468479.

Carstensen, D. W., et al. 2016. Modularity, pollination systems, and interaction turnover in plantpollinator networks across space. - Ecology 97: 1298-1306.

Carstensen, D. W., et al. 2014. Beta diversity of plant-pollinator networks and the spatial turnover of pairwise interactions. - PLoS One 9(11): e112903.

Devictor, V., et al. 2010. Defining and measuring ecological specialization. - Journal of Applied Ecology 47: 15-25.

331 Dormann, C. F. 2011. How to be a specialist? Quantifying specialisation in pollination networks. Network Biology 1: 1-20. 
333 Dunne, J. A. 2006. The network structure of food webs. - In: Pascual, M. and Dunne, J. A. (eds.), 334 Ecological Networks: Linking structure to dynamics in food webs. Oxford University Press, pp. 2733586.

336 Dupont, Y. L., et al. 2011. Scaling down from species to individuals: a flower-visitation network 337 between individual honeybees and thistle plants. - Oikos 120: 170-177.

338 Fox, L. R. and Morrow, P. A. 1981. Specialization: Species property or local phenomenon. 339 Science 211: 887-893.

340 Ibanez, S., et al. 2016. How phylogeny shapes the taxonomic and functional structure of plant341 insect networks. - Oecologia.

342 Junker, R. R., et al. 2013. Specialization on traits as basis for the niche-breadth of flower visitors 343 and as structuring mechanism of ecological networks. - Funct. Ecol. 27: 329-341.

344 King, C., et al. 2013. Why flower visitation is a poor proxy for pollination: measuring single-visit 345 pollen deposition, with implications for pollination networks and conservation. - Methods in 346 Ecology and Evolution 4: 811-818.

347 Morales-Castilla, I., et al. 2015. Inferring biotic interactions from proxies. - Trends Ecol Evol 30: $348 \quad 347-356$.

349 Olesen, J. M., et al. 2011. Missing and forbidden links in mutualistic networks. - Proceedings of the 350 Royal Society B: Biological Sciences 278: 725-732.

351 Ollerton, J. and Cranmer, L. 2002. Latitudinal trends in plant-pollinator interactions: are tropical 352 plants more specialised? - Oikos 98: 340-350.

353 Ollerton, J., et al. 2007. Multiple Meanings and Modes: On the Many Ways to Be a Generalist 354 Flower. - Taxon 56: 717-728.

355 Ollerton, J., et al. 2009. Fly pollination in Ceropegia (Apocynaceae: Asclepiadoideae):

356 biogeographic and phylogenetic perspectives. - Ann Bot 103: 1501-1514. 
Ollerton, J., et al. 2015. Using the literature to test pollination syndromes - some methodological cautions. - Journal of Pollination Ecology 16: 119-125.

Ollerton, J., et al. 2011. How many flowering plants are pollinated by animals? - Oikos 120: 321326.

Poisot, T., et al. 2015. Beyond species: why ecological interaction networks vary through space and time. - Oikos 124: 243-251.

Rosas-Guerrero, V., et al. 2014. A quantitative review of pollination syndromes: do floral traits predict effective pollinators? - Ecology Letters 17: 388-400.

Santamaria, L. and Rodríguez-Girones, M. A. 2007. Linkage rules for plant-pollinator networks:

Trait complementarity or exploitation barriers? - Plos Biology 5: e31. doi:10.1371/journal.pbio.0050031.

Schleuning, M., et al. 2012. Specialization of mutualistic interaction networks decreases toward tropical latitudes. - Current biology : CB 22: 1925-1931.

Silveira, F. A. O., et al. 2016. Ecology and evolution of plant diversity in the endangered campo rupestre: a neglected conservation priority. - Plant and Soil 403: 129-152.

Team, R. D. C. 2008. R: A language and environment for statistical computing. R Foundation for Statistical Computing. Thompson, J. N. 2005. The geographic mosaic of coevolution. - University of Chicago Press. Trøjelsgaard, K., Báez, M., Espadaler, X., Nogales, M., Oromí, P., Roche, F.L. \& Olesen, J.M. (2013) Island biogeography of mutualistic interaction networks. Journal of Biogeography, 40,

378 Trøjelsgaard, K., et al. 2015. Geographical variation in mutualistic networks: similarity, turnover and partner fidelity. - Proc Biol Sci 282. 
380 Tur, C., Vigalondo, B., Trøjelsgaard, K., Olesen, J.M. \& Traveset, A. (2014) Downscaling pollen381 transport networks to the level of individuals. Journal of Animal Ecology, 83, 306-317.

382 Waser, N. M. 2006. Specialization and generalization in plant-pollinator interactions: A historical 383 perspective. - In: Waser, N. M. and Ollerton, J. (eds.), Plant-pollinator interactions: from 384 specialization to generalization. The University of Chicago Press.

385 Waser, N. M., et al. 1996. Generalization in pollination systems, and why it matters. - Ecology 77: $386 \quad 1043-1060$.

387

388

389

390

391

392

393

394

395

396

397

398

399

400

401 
Brazil

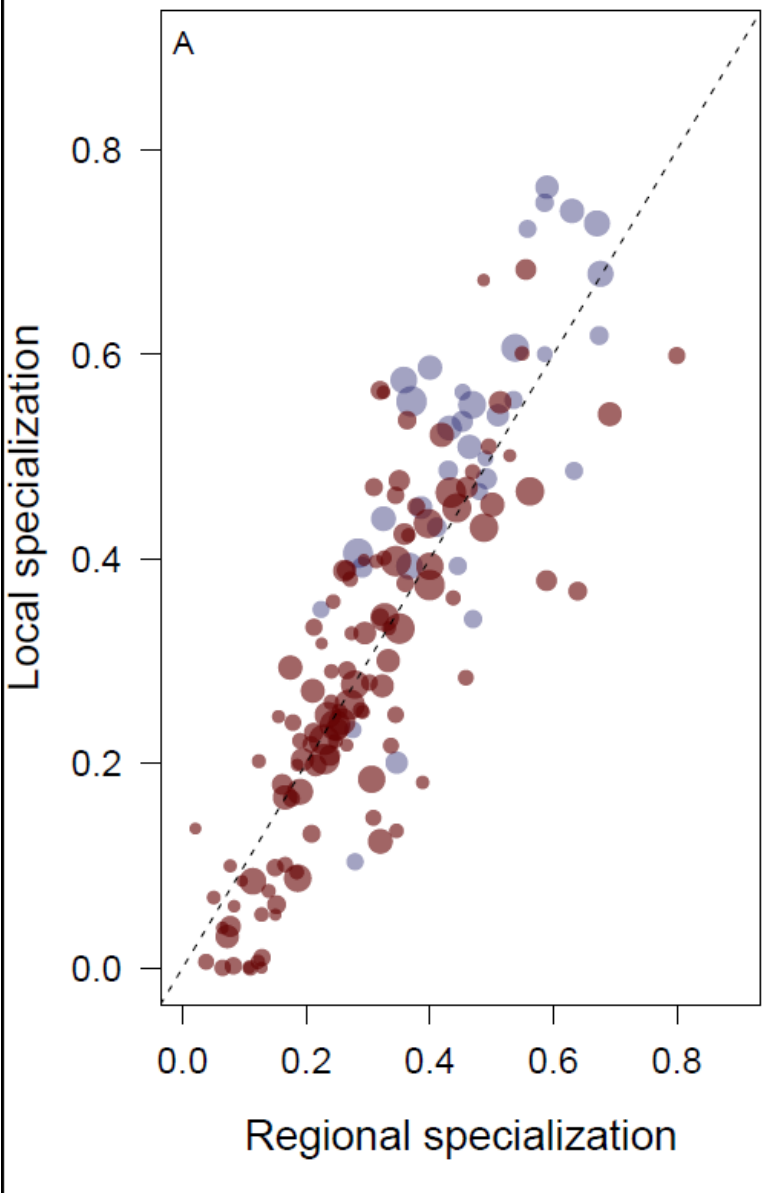

402

403

Fig 1

404
Canary Islands

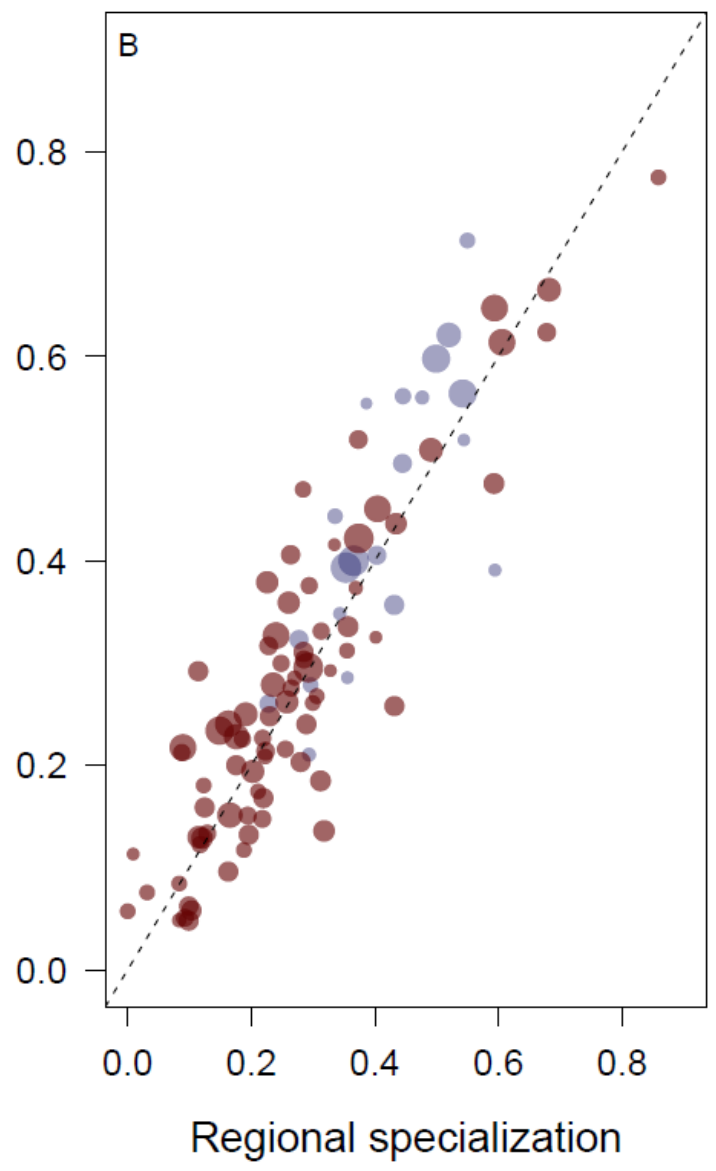



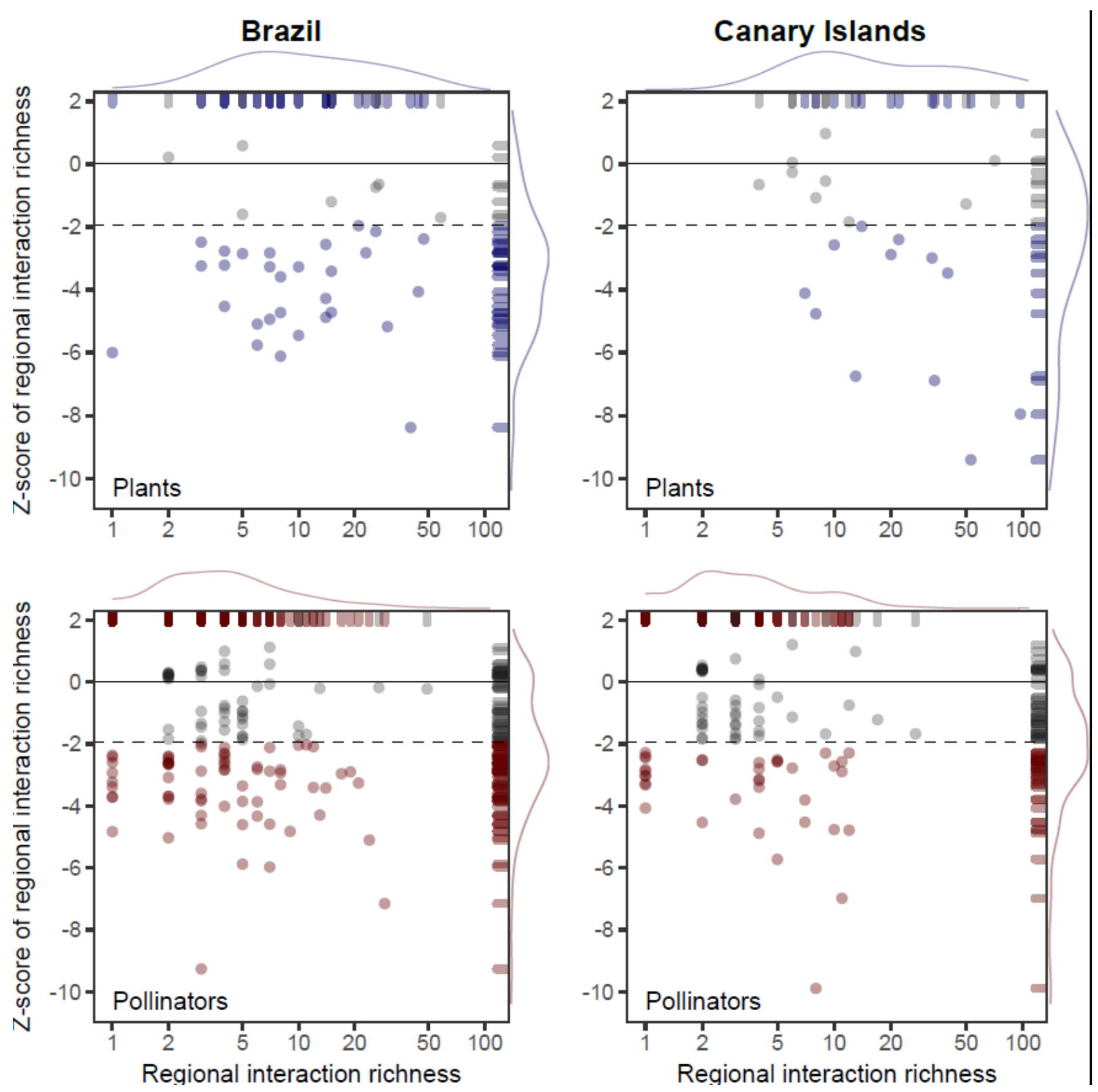

405

406

Fig 2

407 

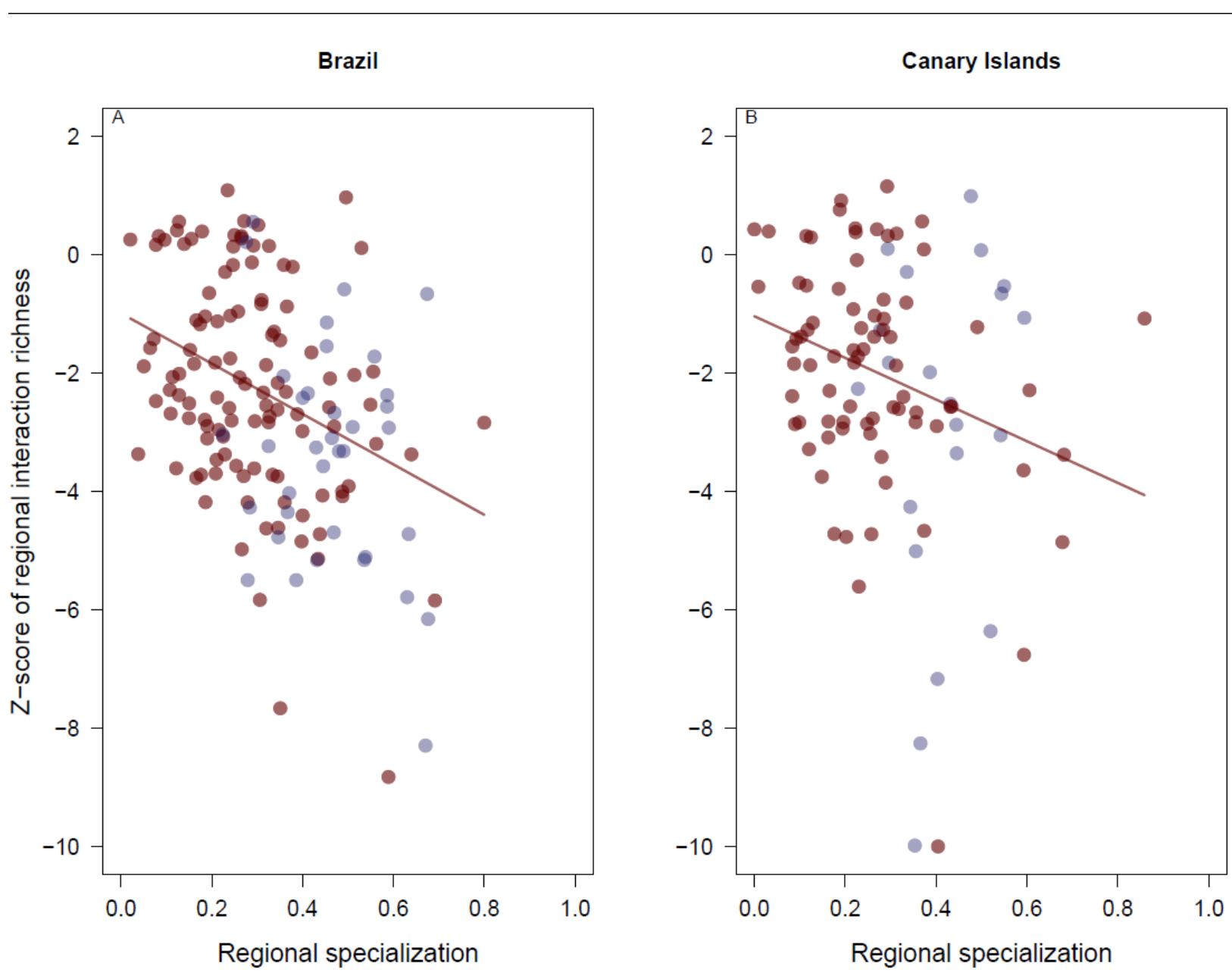

409

Fig 3

410

411

412

413

414

415 